\title{
Sur quelles bases établir des relations stables entre historiens et sociologues?
}

\section{François Chazel}

\section{OpenEdition}

\section{Journals}

Édition électronique

URL : http://journals.openedition.org/ress/371

DOI : 10.4000/ress.371

ISSN : 1663-4446

Éditeur

Librairie Droz

Édition imprimée

Date de publication : 1 mars 2004

Pagination : 63-72

ISBN : 2-600-00941-8

ISSN : 0048-8046

\section{Référence électronique}

François Chazel, «Sur quelles bases établir des relations stables entre historiens et sociologues ?», Revue européenne des sciences sociales [En ligne], XLII-129 | 2004, mis en ligne le 05 novembre 2009, consulté le 19 avril 2019. URL : http://journals.openedition.org/ress/371 ; DOI : 10.4000/ress.371 


\section{SUR QUELLES BASES ÉTABLIR DES RELATIONS STABLES ENTRE HISTORIENS ET SOCIOLOGUES?*}

La question du rapport entre deux disciplines relevant l'une et l'autre des sciences humaines et sociales et, à ce titre, nécessairement voisines, comme l'histoire et la sociologie est, à coup sûr, un thème académique, c'est-à-dire rebattu; mais elle est susceptible d'être abordée sous tant de points de vue divers qu'elle est en même temps inépuisable: elle ne peut jamais être tenue pour close.

Il nous semble, de surcroît, qu'elle revêt aujourd'hui une actualité particulière et ce pour trois raisons principales, dont la première a quasiment un statut d'évidence, la seconde est largement partagée par les observateurs attentifs au développement respectif des deux disciplines et la troisième en revanche n'est, à notre connaissance, guère relevée. Tout d'abord, on ne peut plus - si on l'a jamais pu s'en tenir à un sommaire découpage temporel pour définir le domaine scientifique de l'histoire et de la sociologie: il existe désormais aussi bien une histoire du temps présent qu'une sociologie historique. L'impossibilité manifeste qui en résulte d'enfermer chacune des deux disciplines dans l'étude exclusive d'univers temporels séparés - le passé pour l'histoire, le contemporain pour la sociologie ne fait que reposer avec plus d'acuité la question de leur nature propre, c'est-àdire de leurs différences sur un fond de parenté.

Ensuite, sociologie et histoire ont aujourd'hui - et, espérons-le, définitivement - renoncé l'une et l'autre, même si elles n'ont pas toujours su résister à cette tentation, à l'ambition de constituer la discipline fédératrice des sciences humaines et sociales. Du côté de la sociologie, la conception durkheimienne d'une sociologie appelée à devenir «le système des sciences sociales » ${ }^{1}$ ' a guère été pour les générations ultérieures de sociologues un mot d'ordre efficace. Comme Philippe Besnard l'a bien montré, elle était trop tributaire de l'idée, plus appropriée à un temps d'institutionnalisation de la discipline qu'à sa maturation progressive, de «l'unité du règne social» pour pouvoir être effectivement maintenue ${ }^{2}$. Les sciences sociales particulières ont poursuivi leur développement, à côté des sociologies spécialisées dont l'essor a pourtant été indiscutable. Ces préoccupations

Cet article constitue la version remaniée d'un texte antérieurement publié dans Y. Beauvois et C. Blondel (sous la dir. de), Qu'est-ce qu'on ne sait pas en histoire?, Presses Universitaires du Septentrion, Villeneuve d'Asq (Nord), 1998, p. 117-129.

1 Emile Durkheim et Paul Fauconnet, «Sociologie et sciences sociales» (1903), in Emile Durkheim, Textes 1, Paris, Ed. de Minuit, 1975, p. 121.

2 Voir Philippe Besnard, «L'impérialisme sociologique face à l'histoire», in Historiens et sociologues aujourd' hui, Paris, Editions du CNRS, 1986, p. 27-35, et E. Durkheim et P. Fauconnet, art. cit., p. 158. 
fédératrices ont perduré davantage du côté de l'histoire, à travers les projets intégrateurs de l'école des Annales; mais, à en juger par des travaux récents ${ }^{3}$, elles ne paraissent plus au goût du jour. A partir du moment où il est, comme maintenant, admis que les sciences sociales ne sauraient être ordonnées et organisées autour d'une discipline majeure, qu'il s'agisse de la sociologie ou de l'histoire, chacune de ces dernières se voit reconnue dans son autonomie pleine et entière: leurs liens doivent de ce fait être repensés, indépendamment de tout recours à l'hypothèse facile qui ferait de l'une la «science auxiliaire» de l'autre.

Enfin, il nous parait d'autant plus urgent de revenir, une fois encore, sur l'importance des liens - existants ou à établir - entre histoire et sociologie qu'historiens et sociologues suivent apparemment, depuis peu, des chemins opposés: au risque de formuler en termes trop abruptement contrastés ces orientations franchement divergentes, nous serions tenté d'affirmer que les historiens, à en croire certaines de leurs déclarations de principe, seraient enclins à faire très parcimonieusement appel à la sociologie, si ce n'est à se passer de toute référence à cette discipline, alors qu'a contrario les sociologues, rompant avec une longue période de prévention à l'égard de l'histoire, se montrent plus soucieux de l'ancrage historique des phénomènes sociaux.

La poursuite en histoire de cette stratégie «isolationniste» serait à terme, si notre diagnostic est exact, préjudiciable à l'une et l'autre «sciences»; et c'est pourquoi il nous paraîtrait opportun de jeter les bases d'une collaboration franche, dénuée de tout esprit d'impérialisme, entre historiens et sociologues. Les considérations qui suivent s'inspirent de cet objectif, même si elles ne sauraient, bien sûr, prétendre à le satisfaire.

Pour être en mesure de s'engager franchement sur la voie de cette collaboration, il faut au préalable identifier les obstacles qui sont de nature à la freiner, voire à l'interdire: c'est une première étape dont il est difficile de faire l'économie. Les obstacles en question sont liés avant tout, nous semble-t-il, à certaines conceptions qu'historiens et sociologues se font ou ont pu se faire de leur discipline respective et qui infléchissent plus ou moins fortement l'orientation de leurs recherches.

De la part des sociologues, les ambitions nomothétiques excessives qu'ils ont longtemps affichées pour leur discipline étaient de nature à limiter tout rapprochement avec l'histoire, qui ne pouvait à leurs yeux revendiquer ce statut de science positive. Mais ces ambitions ont été, dans la période récente, revues à la baisse. La sociologie est encore le plus souvent considérée comme une science généralisante, qui vise à établir des régularités (dans la synchronie) et à dégager des tendances (dans la diachronie); elle est même en mesure de formuler des lois. Cependant il ne faut pas se tromper sur la nature de ces lois, qui sont locales et non pas universelles: elles établissent des «relation[s] qui peu[ven]t être observée[s] sous certaines conditions qu'il est impossible de définir de manière exhaustive $»^{4}$; elles sont de la forme:

Nous nous bornerons à citer ici, compte tenu de son caractère synthétique, l'ouvrage dirigé par Jean Boutier et Dominique Julia, Passés recomposés : champs et chantiers de l' histoire, Paris, Ed. Autrement, 1995.

$4 \quad$ Raymond Boudon, La place du désordre, Paris, P.U.F., 1984, p. 87. 


\section{si A, dans certaines conditions (qu'il est impossible de préciser complètement) $B$,}

et sont illustrées, entre autres, par la célèbre proposition d'Alexis de Tocqueville: «Ce n'est pas toujours en allant de mal en pis que l'on tombe en révolution ${ }^{5}$. Il ne paraît pas utile de pousser ici plus loin l'examen; ce bref rappel d'ordre épistémologique suffit à établir la conclusion pertinente pour notre propos: les sociologues ont, dans l'ensemble, renoncé à l'utopie de transcender l'histoire.

En revanche les réticences des historiens à l'égard de la sociologie ont plutôt tendu à s'accroître; et il est piquant de relever que cette prise progressive de distance a pris naissance dans une critique justifiée, même si celle-ci vise davantage une manière de pratiquer l'histoire que la sociologie en tant que telle. Les historiens ont en effet été de plus en plus nombreux à dénoncer les excès d'un certain type d'histoire sociale. C'est ce que fait notamment avec beaucoup de brio Richard Cobb dans La protestation populaire en France, en comparant le Précis d'Histoire de la Révolution française de Soboul à «un ballet historique que l'on pourrait mettre en musique, un pas de deux, et parfois un pas de trois, ... [obéissant à] la règle d'une chorégraphie particulièrement rigoureuse qui ne permettra aucun pas déplacé ${ }^{6}$.

Le malheur, si l'on peut s'exprimer ainsi, c'est que de cette position saine Cobb tire une conclusion fausse le conduisant, selon ses propres termes et par un étrange amalgame, à «un refus catégorique de la sociologie et des chiffres » ${ }^{7}$. Ce refus s'accompagne d'une redéfinition des priorités, en vertu de laquelle il faudrait en histoire redonner à la narration la première place qu'elle n'aurait jamais dû perdre. Cette même orientation est adoptée, de façon encore plus accusée et systématique, par différents auteurs, et notamment par Simon Schama qui, après s'être recommandé explicitement de Cobb, défend dans la Préface à son ouvrage sur la Révolution française l'idée d'un retour à la forme des chroniques du XIX'e siècle ${ }^{8}$.

On est ainsi conduit à se poser une question cruciale: le renoncement - fondé - à une histoire pseudo-systématique et l'importance à nouveau reconnue à la narration entraîneraient-elles l'histoire sur une voie unique qui passerait par la réhabilitation de la chronique? Nous ne le pensons pas, pour notre part; et nous espérons démontrer que l'histoire peut emprunter d'autres voies, à notre sens, plus fécondes.

Avant d'aborder ce versant «positif » de notre argumentation, nous voudrions encore noter une autre raison de l'éloignement relatif des historiens à l'égard de la sociologie. La redécouverte de l'importance du politique, l'attention soutenue accordée aux dimensions culturelles ont amené certains d'entre eux - par exemple le groupe constitué autour de François Furet - à rejeter la vision unilatérale et réductrice des faits historiques, qui privilégiait à l'excès les facteurs sociaux (et

Alexis de Tocqueville, L'Ancien Régime et la Révolution, Paris, Gallimard, 1952, t. 1, p. 223.

Richard Cobb, La protestation populaire en France (1789-1820), trad. fr. de The Police and the People, Paris, Calmann-Lévy, 1975, p. 109-110.

Ibid, p. 13 (note 1).

8 Simon Schama, Citizens : A Chronicle of the French Revolution, New York, Alfred Knopf, 1989, p. XVI. 
socio-économiques). Mais un pas supplémentaire a parfois été franchi: la sociologie a parfois été associée à cette vision unilatérale, comme si elle ne pouvait être d'aucun secours au-delà de la mise en évidence de la composition sociale de tel ou tel groupe ${ }^{9}$ et comme si elle n'avait rien à apporter, dans son registre propre, à l'analyse de la complexité - désormais clairement établie - des faits historiques.

Il convient donc d'une part de redresser cette vision étriquée de la sociologie, d'autre part de faire valoir les droits d'une histoire qui refuserait de s'enfermer dans la chronique, pour sauvegarder les chances d'une collaboration fructueuse entre historiens et sociologues.

Cette collaboration n'implique pas seulement de surmonter des obstacles; elle doit aussi reposer sur une conception des rapports unissant les deux disciplines et, plus précisément, des services qu'elles sont mutuellement susceptibles de se rendre. Elle passe en quelque sorte par une seconde étape, qui consiste à esquisser une perspective générale d'analyse, spécifiant les tâches et les enjeux de l'une et de l'autre. Et il convient, pour ce faire, de renouer avec l'inspiration weberienne.

Ce terme de renouer surprendra peut-être les sociologues; mais il attire l'attention sur un fait souvent négligé: la prépondérance de l'école des Annales, qui a longtemps duré, n'était guère compatible avec la reconnaissance de l'importance centrale de Weber. L'un des fondateurs - à savoir Marc Bloch - était certes un bon connaisseur de l'historiographie allemande mais il fut marqué par l'influence d'un courant tout à fait différent, en l'occurrence celui de Lamprecht. De surcroît cette conception n'a pas prévalu dans l'école des Annales, qui a d'abord maintenu des liens superficiels avec les autres sciences sociales avant de revendiquer pour l'histoire, avec la troisième génération, une sorte d'hégémonie. Le peu d'attirance de Braudel pour Weber est à replacer dans ce contexte: il appartient à une autre tradition de pensée et a défendu un projet qui ne favorisait guère un dialogue constructif avec l'œuvre de Weber.

Ce dernier, s'il distingue fermement la vocation propre de la sociologie, qui «élabore des concepts de types et est en quête de règles générales du devenir » et celle de l'histoire «qui a pour objet l'analyse et l'imputation causale d'actes, de structures et de personnalités individuelles, culturellement importants ${ }^{10}$, insiste immédiatement après sur la profondeur des relations entre sociologie et histoire, d'une manière qui est peut-être de nature à calmer les appréhensions des historiens déçus par une définition de l'histoire qui souligne son aspect idiographique. La sociologie ne se contente pas en effet de puiser ses matériaux dans des réalités significatives du point de vue historique, «elle élabore ses concepts et en recherche les règles avant tout également $d u$ point de vue de la possibilité de rendre service à l' imputation causale historique des phénomènes importants pour la culture $\rangle^{11}$. Weber précise la nature de ce service en ajoutant que de tels concepts sont sans doute pauvres en contenu par rapport à la réalité mais que leur

Dans maints ouvrages d'historiens, l'analyse «sociologique» se réduit en effet à l'examen de la composition sociale du parti, du mouvement ou du groupement sur lequel porte, en totalité ou en partie, la recherche.

10 Max Weber, Economie et société, Paris, Plon, 1971, p.17. Les termes soulignés sont en italique dans le texte.

${ }_{11} \quad$ Ibid., p. 17. 
fécondité provient de leur «univocité accrue » ${ }^{12}$, sur laquelle se fonde la démarche interprétative.

Si l'on va maintenant au-delà des particularités et des subtilités du type idéal dont il recommande ici l'usage, il paraît permis de conclure que Weber invite plus généralement le sociologue à construire des modèles d'analyse qui ne doivent pas être confondus avec la réalité mais qui sont à même de l'éclairer et peuvent être, le cas échéant, partagés avec l'historien.

Conformément à cette orientation, il reviendrait au sociologue d'isoler analytiquement des dimensions et peut-être aussi, comme nous aurons l'occasion de le signaler, des processus.

Si l'on accepte cette orientation générale, il reste encore - et cette troisième étape n'est sans doute pas la plus facile - à la mettre en œuvre dans le cadre d'une démarche rigoureuse. Nous nous bornerons ici à dégager certains des impératifs auxquels elle doit satisfaire.

Il nous semble, tout d'abord, que la visée comparative est une composante essentielle de tout programme de recherche ambitieux. Nous n'entendons nullement dire par là qu'il faille dans tous les cas procéder à des comparaisons systématiques: celles-ci ne sont pas toujours possibles, compte tenu des données disponibles; et leur importance varie, selon l'objet de recherche. Mais il est en revanche crucial d'adopter un cadre de référence comparatif, qui autorise des comparaisons partielles sur des dimensions jugées significatives par le chercheur.

On ne peut manquer de rappeler ici - et une fois encore - la maîtrise de Tocqueville dans le maniement de la comparaison, tout autant implicite qu'explicite: la société française constitue en quelque sorte l'arrière-plan sur lequel se détachent à la fois les vertus et les faiblesses de la démocratie américaine, tout comme l'Angleterre libérale du XVIII ${ }^{\mathrm{e}}$ siècle représente le contrepoint de la France de l'Ancien Régime engagée sur les voies périlleuses de la centralisation et de la révolution. On peut également se référer, pour prendre une illustration moins classique, aux articles - peu connus - de Max Weber sur la Révolution russe de 1905-1906 ${ }^{13}$. Dans cet exemple aussi, l'analyse est - indirectement éclairée par un jeu de comparaisons, qui s'organise autour de deux pôles distincts: d'une part, du côté du «mouvement » révolutionnaire, le cas russe est confronté à un modèle de révolution libérale, tel qu'il est illustré dans ses premières phases et dans ses résultats par la Révolution française; d'autre part, considéré cette fois du point de vue gouvernemental, le même cas est rapproché de l'Empire allemand, plus exactement de ses stratégies politiques conservatrices et de ses problèmes pendant la période wilhelminienne. Il faut souligner que cette double référence comparative n'implique aucunement une méconnaissance des spécificités de la situation russe; elle permet en revanche de voir dans la Révolution de 1905 non pas une préparation à une révolution ultérieure (à savoir la Révolution de 1917)

12 Ibid., p. 17. Le terme d'univocité est en italique dans le texte.

13 Max Weber, «Zur Lage der bürgerlichen Demokratie in Russland» (1906) et «Russlands Übergang zum Scheinkonstitutionalismus » (1906), in Max Weber Gesamtausgabe, I (Schriften und Reden), 10, Zur Russischen Revolution von 1905, Tübingen, J.C.B. Mohr, 1989, p. 86-279 et 293 679. On relèvera que le grand spécialiste de Weber, Wolfgang J. Mommsen, a assumé la responsabilité de ce volume, avec le concours de D. Dahlmann. 
mais un essai de révolution libérale qui est précisément condamné à l'échec par les particularités russes comme par le déroulement même du processus révolutionnaire.

Peut-être certains historiens ne voudront-ils, à partir de ces exemples, voir dans notre position que la défense d'une méthode qui fait naïvement appel aux analogies. Ils ne feraient par là que reprendre à leur compte une tradition - bien implantée dans leur discipline - de suspicion à l'égard des analogies, tenues pour incertaines, et, par voie de conséquence, à l'égard même de toute comparaison dont elles sont l'instrument. Ainsi Seignobos considère que les «phénomènes suffisamment analogues pour permettre une comparaison» sont quasi exceptionnels, «qu'il n'y a jamais de similitudes complètes » dans le champ de l'histoire et que «des analogies vraiment précises entre deux séries» de phénomènes historiques ne s'offrent que rarement à l'historien ${ }^{14}$. Seignobos se montre ici trop exigeant; ou, plus précisément, il nous paraît tirer d'un constat - celui du caractère irréductible des spécificités - une conclusion excessive et, à notre sens, non fondée sur la validité de la méthode comparative qu'il juge limitée ${ }^{15}$.

En fait la comparaison n'implique nullement, pour être pertinente, l'établissement d'homologies, qui sont rarissimes. Il faut même se garder de la tentation de postuler des homologies, là où on ne peut établir que des analogies, c'est-à-dire discerner ce que des phénomènes globalement distincts peuvent avoir de commun. L'usage tempéré des analogies confère, nous semble-t-il, toute sa portée aux comparaisons et c'est pourquoi l'adoption d'un cadre de référence comparatif est, à notre sens, essentielle pour l'étude socio-historique, que celle-ci prenne la forme de l'histoire comparée ou d'une sociologie attentive à l'inscription des phénomènes dans l'histoire.

L'histoire comparée partage avec la sociologie « historique» un autre trait: elle a besoin d'un éventail de concepts pertinents, qui est une sorte de fonds plus ou moins commun aux deux disciplines, même si elles n'en font pas le même usage. Une des tâches majeures de la sociologie consiste précisément à élaborer des jeux de concepts et à les mettre à la disposition d'autres sciences sociales, en particulier de l'histoire. Certes on peut admettre avec Pipes que pour l'historien «les catégories utilisées en vue de l'analyse des faits concernant une nation donnée doivent dériver en premier lieu de la propre histoire intérieure de cette nation ${ }^{16}{ }^{16}$, mais à condition d'ajouter que de telles catégories ne sauraient suffire, même à l'appréhension de la singularité qui appelle, comme on vient de le voir, une analyse en termes à la fois de proximité et de différences. Elles doivent être complétées par des concepts transversaux, si l'on peut user de cette métaphore

Ces formulations sont tirées d'un débat qui a suivi un exposé de Seignobos sur «L'inconnu et l'inconscient en histoire » devant la Société française de philosophie (1908) et qui a notamment donné lieu à une confrontation entre les vues de Durkheim et celles de Seignobos. C'est pourquoi ce débat a été partiellement reproduit dans Emile Durkheim, Textes 1, p. 199-217; les propos de Seignobos que nous avons cités se trouvent aux pages 207-208.

15 Vis-à-vis du scepticisme de Seignobos, l'optimisme durkheimien sur les «vertus » de la comparaison nous paraît avoir quelque chose de salutaire, même s'il convient de le tempérer.

16 Richard Pipes, «Max Weber et la Russie (II)», Le Contrat social, 1960, IV, p. 153. Le reproche que Pipes adresse sur ce plan à Weber mérite, pour le moins, d'être nuancé. 
pour désigner des notions applicables à une pluralité de sociétés, voire, dans de multiples cas, à plus d'un type de sociétés. Il est vrai que leur usage comporte des risques - d'anachronisme pour l'historien, de transposition abusive selon le sociologue - mais il est requis par tout essai d'interprétation.

Il ne nous est pas possible, dans le cadre de cet article, de nous étendre davantage sur ce point, si ce n'est pour préciser que l'outillage conceptuel proposé par la sociologie ne concerne pas seulement, selon une image assez répandue chez les historiens, les classifications sociales, les modes de stratification et les divers ordres de groupements. Il porte également sur les «normes», les «valeurs» et les «croyances» ou encore sur les «représentations » et les «idéologies ${ }^{17}$, c'est-àdire justement sur les dimensions normatives et culturelles dont l'étude est aujourd'hui au cœur des recherches de nombreux historiens, qui souvent ne soupçonnent guère l'intérêt d'un éclairage sociologique en ces domaines.

Il ne suffit pas, pourtant, d'adopter un cadre de référence comparatif et de disposer de grilles conceptuelles élaborées - qui ne se réduisent pas, notons-le en passant, à de simples schèmes classificatoires - pour fonder une démarche rigoureuse: celle-ci resterait en quelque sorte inachevée si elle n'aboutissait pas à la formulation de véritables modèles d'analyse. Plutôt que de nous en tenir à des recommandations générales sur le sujet, nous nous risquerons ici, sinon à en proposer un, tout au moins à esquisser un mode d'approche des phénomènes révolutionnaires.

Du côté de la sociologie, s'est développé, en particulier dans les pays anglosaxons, un courant de pensée construit autour de deux axes: l'établissement des causes des révolutions et l'élucidation de leurs conséquences. Comme on le voit, cette perspective vise à appréhender le phénomène dans sa globalité; et elle n'accorde qu'une place secondaire, pour ne pas dire mineure, à l'événement luimême. Il n'est donc pas surprenant qu'elle ait pu être qualifiée de «structurelle» par l'auteur qui l'a prônée, à savoir Theda Skocpol ${ }^{18}$.

Nous voudrions suggérer une autre voie, qui implique, croyons-nous, un partage différent des tâches entre sociologie et histoire. A nos yeux, la grande faiblesse du raisonnement en termes de causes et de conséquences est de laisser de côté les processus révolutionnaires; ceux-ci deviennent plutôt, à partir du moment où ils restent en marge de l'effort interprétatif, la matière privilégiée de chroniques narratives, qui constituent en quelque sorte la nécessaire contrepartie, si ce n'est l'envers, de ce type de sociologie «historique». La sociologie ne doit pas, à notre sens, rester muette sur les processus sous-jacents aux révolutions; c'est au contraire en partant de leur analyse que le sociologue peut, à sa manière, aider à penser la dynamique révolutionnaire. Mais, pour être féconde, une telle entreprise requiert de renoncer aux facilités de l'appréhension globalisante de phénomènes

${ }_{17}$ Nous nous bornerons ici à citer la seule contribution de Clifford Geertz (The Interpretation of Cultures, New York, Basic Books, 1973, chap. VIII), moins en raison de son incontestable originalité, avec son insistance sur les «cadres symboliques» de l'idéologie, que parce qu'elle nous offre l'occasion de rappeler que la sociologie et l'anthropologie culturelle partagent les mêmes ambitions conceptuelles et les mêmes enjeux théoriques, tout en différant par leurs objets.

18 Etats et révolutions sociales: La révolution en France, en Russie et en Chine, Paris, Fayard, 1985 (éd. orig. : 1979), p. 34, 40. 
aussi complexes: il convient de les décomposer en dimensions analytiquement isolables et c'est sur elles, prises séparément, qu'il faut faire prioritairement porter l'examen.

L'une d'entre elles mérite tout particulièrement de retenir l'attention: c'est la mobilisation, saisie comme processus, voire comme ensemble de processus. Toute «grande» révolution «passe» par des mobilisations collectives, qui ne se laissent pas seulement appréhender sur le rythme irrégulier des journées révolutionnaires et qui revêtent, du point de vue de la participation populaire, des formes comme une intensité variables. Les «insurrections» paysannes notamment doivent être envisagées sous l'angle de la mobilisation; et il ne suffit pas à cet égard de spécifier les conditions favorables à leur émergence, comme le fait Skocpol, il est aussi nécessaire de les embrasser dans l'intégralité de leur développement, avec l'objectif de dégager la nature de ce mode de mobilisation et non pas simplement d'en décrire le déroulement ${ }^{19}$. L'éclaircissement progressif des processus mêmes de mobilisation permettrait de mieux cerner leur contribution à la dynamique révolutionnaire, en termes comparés comme dans des cas spécifiques, et d'aller ainsi au-delà d'une reconnaissance - trop générale pour être satisfaisante - de leur importance.

L'idéologie constitue une autre dimension qui appelle un examen attentif. Plus exactement, ce qu'il faudrait saisir, dans notre perspective, c'est le rôle qu'elle est susceptible d'avoir dans la dynamique révolutionnaire elle-même. On est ici, bien sûr, très éloigné de l'imagerie qui, conformément à une vision naïvement volontariste de la révolution, postule au départ un projet idéologique plus ou moins cohérent, dont il resterait à étudier le degré de réalisation ou par rapport auquel, selon les «réalistes »ou les dissidents, on n'aurait qu'à observer les déviations effectives. Mais on voudrait aussi aller au-delà de la conception, infiniment plus solide et brillamment illustrée, qui insiste sur la fonction préparatoire de l'idéologie, sur son rôle de ferment en quelque sorte, qu'on le juge pernicieux ou bénéfique. Sur ce plan du moins, la source d'inspiration ne serait pas prioritairement tocquevillienne; le modèle serait plutôt à tirer de Cochin mais d'un Cochin épuré de ses biais. La force de Cochin - et la fascination qu'il a pu exercer, notamment sur François Furet - vient de ce qu'il inscrit l'idéologie au cœur même de l'enchaînement des processus révolutionnaires. Sa faiblesse tient à un enracinement trop marqué dans une tradition de pensée contre-révolutionnaire; la célèbre proposition: «Où la liberté règne, c'est la machine qui gouverne » n'est pas acceptable; il serait sans doute plus exact de dire que l'idéologie de la communauté transparente favorise l'avènement de la dictature d'une minorité ${ }^{20}$. De surcroît, Cochin met à l'œuvre une logique inexorable, là où l'image la plus appropriée serait plutôt celle d'une ligne brisée: l'enchaînement ne prend pas nécessairement la forme d'un «engrenage»; la dimension idéologique ne peut être appréciée indépendamment

19 L'ouvrage de John Keep, The Russian Revolution: A Study in Mass Mobilization, New York, Norton, 1976, en reste justement à ce niveau descriptif.

20 Nous avions déjà formulé la même idée dans notre article «Idéologie et processus révolutionnaires», Revue française de sociologie, 1989, XXX, p. 431-453. Le thème de la communauté transparente, sa signification et sa portée sont bien mis en évidence par Lynn Hunt dans son ouvrage Politics, Culture and Class in the French Revolution, Berkeley, University of California Press, 1984. 
des enjeux de pouvoir et des solutions momentanées qu'ils reçoivent: son poids varie avec eux, dans la mesure où il est tributaire de ces luttes en vue de l'établissement d'une légitimité politique. On est ainsi amené à renoncer à la thèse qui ferait du jacobinisme la vérité ultime du mouvement révolutionnaire.

A chacune des deux grandes dimensions qui viennent d'être présentées peuvent être consacrés des programmes entiers de recherche. Mais notre projet trouverait son véritable aboutissement si, en croisant ces dimensions entre elles et avec une troisième - les enjeux du pouvoir - qui a été simplement mentionnée, on entreprenait l'élaboration d'un modèle multidimensionnel visant à cerner de plus près la dynamique révolutionnaire.

Cet effort pour comprendre la dynamique révolutionnaire impose une priorité de l'analyse par rapport au récit et donc le refus de la chronique, dont l'éventuelle séduction littéraire cache mal la pauvreté interprétative; il ne peut non plus s'accommoder d'un cadre d'analyse structurel, qui méconnaît la spécificité même du phénomène révolutionnaire en négligeant les processus et leur enchaînement. Nous tenons également à préciser - et sur ce point nous nous séparons de François Furet - qu'à notre sens une telle perspective n'implique pas une sorte de revanche du politique sur le social: certes il convient, avec les plus éminents des spécialistes contemporains, de reconnaître au politique la place centrale qui lui revient dans les révolutions mais, pour parler momentanément le langage des historiens, l'histoire politique ne peut faire l'impasse sur celle de la société. On ne peut comprendre pleinement l'impact du discours révolutionnaire si on le dissocie des pratiques d'association et de réunion qui lui ont donné naissance, en particulier dans le cas de la Révolution française ${ }^{21}$.

Ce programme peut paraître présomptueux; pour notre part, nous serions plutôt enclin à en tirer la conclusion que sa réalisation n'est envisageable que sur la base d'une profonde collaboration entre historiens et sociologues. Nous avons ici soutenu l'idée que les sociologues avaient leur mot à dire dans l'analyse des processus révolutionnaires; mais ils ont besoin de la solidité du travail historique, et ce d'autant plus que ce terrain est en grande partie neuf pour eux. Les historiens seront sans doute plus réticents à accepter le concours des sociologues; peut-être ceux-ci pourraient-ils, selon notre vision optimiste, les aider à adopter une approche ambitieuse, tournée vers l'interprétation des processus plutôt que vers un essai de restitution de l'événement.

Si souhaitable soit-elle, une collaboration stable entre historiens et sociologues, notamment dans le domaine de l'étude des révolutions, ne peut s'instaurer qu'à deux conditions : la première suppose que les historiens, ou tout au moins une fraction significative parmi eux, privilégient une conception et une pratique de leur discipline relevant de ce que l'on appelle souvent l'histoire interprétative; la seconde implique que la sociologie, dans ses usages effectifs comme dans l'idée que s'en font les historiens, ne soit pas réduite à une spécialité traitant de la stratification sociale et des groupements. Précisons que nous ne voudrions pas par là en donner une image édulcorée mais bien plutôt lui conférer une assise plus large: à ce titre, elle est en mesure d'apporter un éclairage sur le politique ainsi que sur le culturel, ce que visaient à suggérer, sinon à établir, les pages précédentes.

${ }^{21}$ Nous partageons sur ce point l'appréciation de Lynn Hunt. 
Ces conditions sont loin d'être toujours satisfaites; mais elles ne représentent pas non plus un idéal inaccessible. Entre une histoire interprétative, dégagée des excès de l'histoire pseudo-systématique et soucieuse de dépasser les fausses évidences de la chronique, et une sociologie ouverte sur la comparaison, engagée dans la production d'outils conceptuels et davantage préoccupée de modèles que de lois pourrait se développer une collaboration féconde, plus suivie qu'elle ne l'a généralement été au cours du siècle passé: c'est en tout cas la conviction que nous avons tenu à exprimer et cherché à défendre au cours de ce texte.

Université de Paris IV - Sorbonne

francois.chazel@paris4.sorbonne.fr 